\title{
Concealed Paroxysmal Atrioventricular Block: Diffuse Congenital Atrioventricular Conduction System Disorder with Nonpropagated His Bundle Depolarizations
}

\author{
MACDONALD DICK II, ROBERT H. BEEKMAN, \\ and CAROL H. KASTEN-SPORTES \\ From the Division of Pediatric Cardiology, C.S. Mott Children's Hospital, and the Department of \\ Pediatrics, University of Michigan, Ann Arbor, Michigan
}

DICK, M., II, ET AL.: Concealed paroxysmal atrioventricular block: diffuse congenital atrioventricular conduction system disorder with nonpropagated His bundle depolarizations. A 2 1/2-year-old girl with bradycardia and left bundle branch block at birth began to experience "night cries" when deeply asleep. Electrophysiological study demonstrated congenital diffuse atrioventricular conduction disease with concealed paroxysmal atrioventricular block, nonpropagated His bundle depolarizations, severe sinus node abnormality, and a low atrioventricular junctional escape rhythm with probable reciprocation. After pacemaker implant, the "night cries" ceased. (PACE, Vol. 11, September 1988)

atrioventricular node, complete heart block, paroxysmal atrioventricular block, electrophysiology, electrocardiography

\section{Introduction}

Complete interruption of atrioventricular conduction in children is most often congenital in origin or surgically induced; it is usually permanent, and regardless of mode or time of onset, it has been localized by His bundle recordings within the atrioventricular node (i.e., above the bundle of His, supraHisian), within the His-Purkinje system (below the bundle of His, infraHisian) or within the bundle of His itself (intra-Hisian). ${ }^{1}$ Propagation of the cardiac impulse through the atrioventricular node and/or His-Purkinje system may be altered or even completely and paroxsymally interrupted by transient changes in vagal tone and/or basic cycle length, as well as the occurrence of tachycardia - dependent (phase

\footnotetext{
Address for reprints: Macdonald Dick II, M.D., Division of Pediatric Cardiology, Department of Pediatrics, C.S. Mott Children's Hospital, Room F1126, Box 0204, University of Michigan Medical Center, 1500 E. Medical Center Drive, Ann Arbor, MI 48109-0204, USA.
}

Received July 2, 1987; revision October 19, 1987; accepted December 24, 1987.
3) block, bradycardia-dependent (phase 4) block, or concealed extrasystoles (pseudo-atrioventricular block). ${ }^{2-7}$ In this report we outline the unique clinical, electrocardiographic, and electrophysiological findings in a 21 / 2 -year-old girl with periods of asystole during sleep. However, because of the absence of sinus node activity, no regular sinoatrial $\mathrm{P}$ waves were present on the surface electrocardiogram; electrophysiological study was necessary to demonstrate that the mechanism of the pauses was paroxysmal atrioventricular block concealed from the surface recording.

\section{Case Report}

The patient weighed $3.4 \mathrm{~kg}$ at birth. Gestation was complicated by apparent (culture negative) maternal genital herpes at 6 weeks of gestation; the lesions cleared within 10 days. At 4 months of gestation maternal proximal interphalangeal pain and tenderness, accompanied by an increased erythrocyte sedimentation rate and hypergammaglobulinemia appeared; the symptoms spontaneously resolved. A maternal anti-nuclear antibody 
ular block is unclear. Because of probable reciprocation within the atrioventricular node (see below), relatively early His bundle repolarizations were present. Review of the His bundle recordings demonstrated that $90 \%$ of His bundle depolarizations with coupling $(\mathrm{HH}$ or $\mathrm{VH})$ intervals of $470 \mathrm{~ms}$ or less and all beats with coupling intervals of $410 \mathrm{~ms}$ or less failed to propagate to the ventricles presumably because of phase 3 (tachycardia-dependent) block. However, although we were able to detect this evidence for phase 3 block, as well as for normal H-V conduction over an intermediate range of coupling intervals, we could not find a third longer cycle length range at which atrioventricular block occurred. Thus, no evidence for phase 4 (bradycardia-dependent) block could be found. Therefore, the most likely mechanism of the observed asystole in this patient is concealed paroxysmal atrioventricular block within the right bundle branch mediated through the profound parasympathetic influence of sleep.

Two other levels of abnormality within the cardiac conduction system could be identified. Sinus node abnormality was indicated both by the sinus bradycardia $(80 \mathrm{bpm})$ (Fig. 1A) present at birth, and the progression to disappearance of sinus (high-to-low) P waves on the surface electrocardiogram and at electrophysiological study.

The third level of abnormality was located within the atrioventricular node. The dominant rhythm was an escape ectopic focus postulated to arise, because of the observed low-to-high atrial activation sequence (Fig. 3), in the low atrioventricular junction at a cycle length of 740-1000 ms (Figs. 3-5). Both during surface ECG monitoring and electrophysiological study there was repetitive group beating such that the third or fourth His bundle discharge recurred at constant intervals of $460-470 \mathrm{~ms}$. This pattern of grouped beating can be explained by several mechanisms. At very slow discharge rates subsidiary pacemakers in the rabbit atrioventricular node ${ }^{10}$ the rabbit atrioventricular valve leaflet ${ }^{11}$ and the canine

\section{References}

1. Simon, JB, Dick M, Stern AM, et al. Ventricular pacing in children. Pace 1982; 25:836-844.

2. Zipes DP. Specific Arrhythmias: Diagnosis and Treatment. In Braunwald E (ed): Heart Disease. atrioventricular junction ${ }^{12}$ have been shown to exhibit very slow conduction into adjacent tissue exit block and, with autonomic manipulation, automaticity, and even reciprocation. Although enhanced abnormal automaticity cannot be entirely excluded to explain the grouped beating in our patient with this very slow escape pacemaker rate, the fixed coupling led us to postulate reciprocation within a markedly abnormal atrioventricular node. In short, the second (or third) impulse during slow retrograde conduction reciprocated within the middle and/or high atrioventricular node to return to low atrial nodal region and discharge the His bundle at a fixed interval. Observations supporting this interpretation of the different levels of reciprocation and block within the atrioventricular node have been reported, using deductive reasoning, from surface human electrocardiograms ${ }^{13}$ as well as from the above cited experimental models. ${ }^{10,11}$

The etiology of the atrioventricular conduction system disorder in this child is unknown. The clinical course and the laboratory findings are not convincing of either a maternal collagenvascular disorder, myocarditis, or an intrauterine infection. Because sinus node activity was absent, the escape cardiac rhythm was junctional. The periods of asystole at night could be interperted from the surface electrocardiogram as a pause in the discharge rate of the junctional pacemaker. However, electrophysiological study demonstrated that the cause of the asystole and "night cries" was paroxysmal atrioventricular block distal to the His bundle and concealed from the surface electrocardiogram. The progressive findings in this $2 \frac{1}{2}$-year-old-girl strongly indicated a congenital atrioventricular conduction system disorder and represents an example of concealed paroxysmal atrioventricular block.

Acknowledgment: The authors gratefully acknowledge the helpful review of the original electrophysiological tracings by Drs. Grace Wolff and Augustin Castellanos, Jr., as well as the excellent secretarial assistance of Ms. Colleen Rauch.

Philadelphia, WB Saunders Co., 1984, pp. 730-735.

3. Strasberg B, Lam W, Swiryn S, et al. Symptomatic spontaneous paroxsymal AV node block due to 
localized hyperresponsiveness of the AV node to vagotonic reflexes. Am Heart J 1982; 103:795-801.

4. Rosen KM, Rahimtoola SH, Gunnar RM. Pseudo AV block secondary to premature non-propagated His bundle depolarizations: Documentation by His bundle electrocardiography. Circulation 1970; 42:367-373.

5. Rosenbaum MB, Elizari MV, Levi RI, et al. Paroxysmal atrioventricular block related to hypopolarization and spontaneous diastolic depolarization. Chest 1973; 63:678-688.

6. Castellanos A, Kluiddus SA, Sommer LS, et al. His bundle recordings in bradycardia - dependent AV block induced by premature beats. Br Heart J 1975; 37:570-575.

7. Denes P, Murabit I, Ezri M, et al. Tachycardia and bradycardia dependent atrioventricular block: Observations regarding the mechanism of block. J Am Coll Cardiol 1987; 9:446-449.

8. Dick M, Campbell RM. Advances in the management of cardiac arrhythmias in children. Ped Clin North Am 1984; 31:1175-1195.
9. Dick M, Byrum CJ, Hees P, et al. Effect of morphine, benedryl, and chloral hydrate on the sinus node in the early post-operative cardiac patient. Ped Res 1982; 16:98A.

10. Watanabe Y, Driefus LS. Levels in concealment in second-degree and advanced second-degree AV block. In Driefus LS, Likoff W (eds): Cardiac Arrhythmias. New York, Grune and Stratton, 1973, pp. 305-324.

11. Rozanski G, Jalife J. Automaticity in AV valve leaflets of rabbit heart. Am J Physiol 1986; 250:H397406.

12. Urthaler F, Neely BH, Hageman GR. Atrioventricular junctional tachycardia during heart block. J Am Coll Cardiol 1986; 8:657-660.

13. Pick A, Langendorf R. Specific mechanisms of various disorders of impulse formation, conduction, and their combinations. In Pick A, Langendorf R (eds): Interpretations of Complex Arrhythmias. Philadelphia: Lea and Febiger, 1979, p. 572. 